\title{
Isolated Dislocation of the Distal Radioulna Joint: An Unrecognized Emergency
}

\author{
Ralahy Fanjalalaina, Randimbinirina Zakarimanana, Andrianimaro Florelia, Rohimpitiavana Amboara, \\ Razafimahandry Henri
}

Ministry of Public Health Ringgold Standard Institution, Fianarantsoa, Madagascar

Correspondence to: Dr. Ralahy Fanjalalaina; Email: fafah.ralahy@gmail.com.

\section{Summary}

Dislocation of the isolated distal radioulna joint (DRUJ) is rare. The clinical and radiological signs are not suggestive, making diagnosis difficult and sometimes late. Care remains disparate for both recent and neglected forms. To illustrate the management of these lesions in light of data from literature, we report two cases of isolated dislocation of the distal radioulna joint. The first patient was a 22year-old woman who presented with a recent palmar traumatic dislocation of the left DRUJ whose reduction followed by immobilization restored the function and mobility of the wrist. The second patient was a 34-yearold man with dorsal dislocation from the DRUJ who received Sauvé-Kapandji intervention, with a marked improvement in wrist mobility. DRUJ dislocations must be diagnosed and reduced early as neglected or recurrent lesions require surgery. For this case, intervention with Sauvé-Kapandji had a good result.

Keywords: Dislocation, Distal Radioulna, SauvéKapandji, Surgery, Wrist

Ann Afr Surg. 2020; 17(2):93-96

DOI: http://dx.doi.org/10.4314/aas.v17i2.12

Conflicts of Interest: None

Funding: None

(C) 2020 Author. This work is licensed under the Creative Commons Attribution 4.0 International License.

\section{Introduction}

Distal radioulna dislocation is usually associated with fracture of the forearm bones. First described by Desault (1), isolated distal radioulna dislocation is a rare clinical entity. The mechanism is always traumatic and consists of a strong supination of the forearm for palmar dislocation, or a strong pronation for dorsal dislocation (2). The direction of dislocation is defined by the position of the ulna head with respect to the distal radius (3). Care remains controversial for both recent and neglected forms. Some authors routinely recommend surgery $(1,4)$ while others suggest nonoperative therapy $(5,6)$. The aim of this work is to illustrate the management of isolated distal radioulna dislocations in light of data in literature by reporting two clinical cases.

\section{Case 1}

A 22-year-old student consulted at the emergency department after a left wrist injury in a sports accident followed by a fall, on her outstretched left arm with the wrist in supination. The patient complained of severe pain in her wrist associated with functional impairment. The examination showed distortion of the left wrist with loss of the medial concavity, giving way to an asymmetrical concavity compared with the contralateral side. X-ray of the wrist and left forearm face and profile showed palmar dislocation of the distal radioulna joint without associated fracture (Fig. 1).

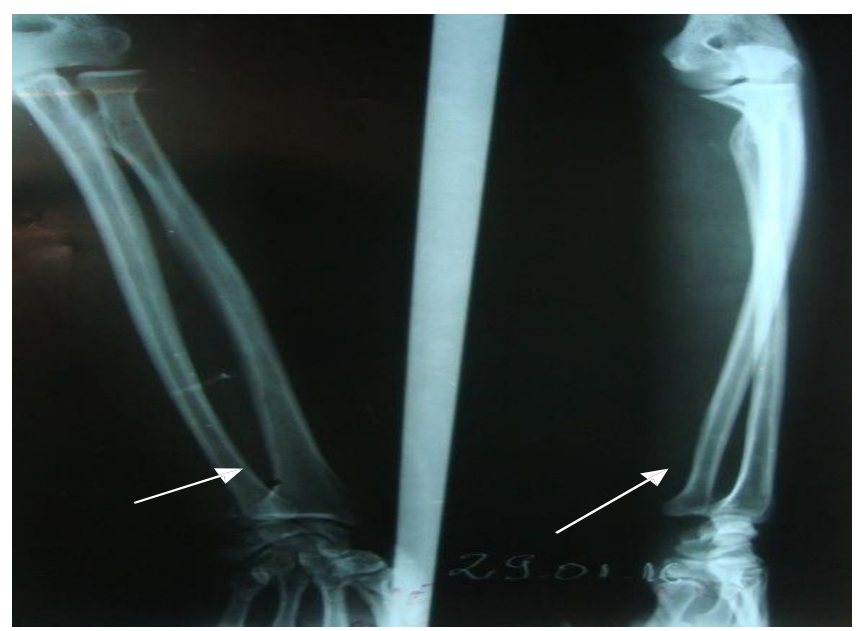

Figure 1: Radiograph of the left forearm in frontal and lateral incidence showing palmar dislocation of the DRU. 
A closed reduction under general anesthesia was performed followed by a brachio-antebrachio-palmar cast in neutral position for 3 weeks (Fig. 2). The elbow was released on the third week and the wrist joint was kept immobilized for three additional weeks. After 2 weeks of rehabilitation, she resumed her daily activities without difficulty. After 2 years, no recurrence of luxation was noticed.

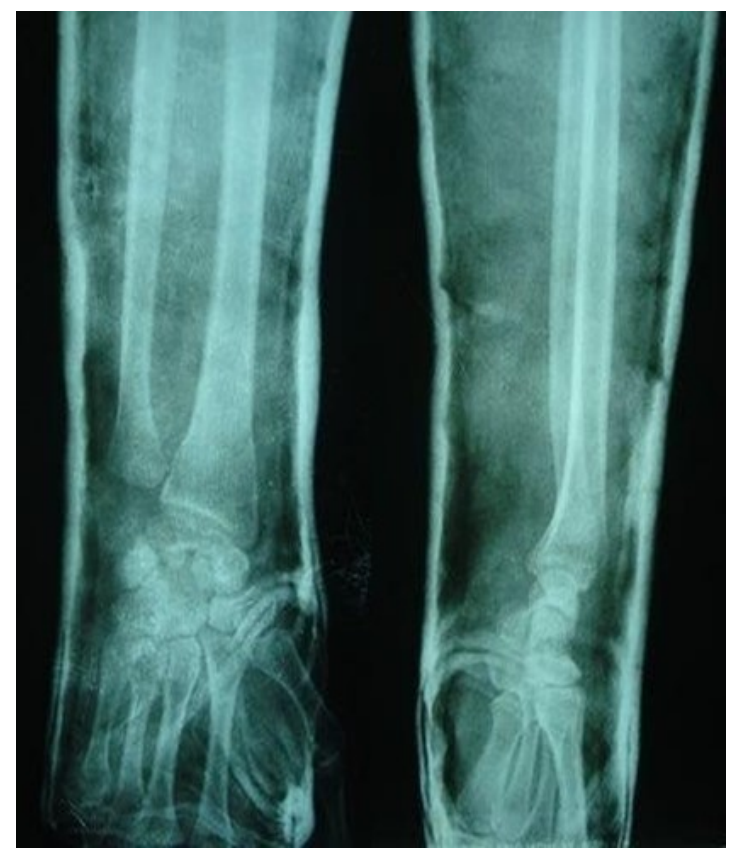

Figure 2: Control radiograph centered on the left wrist in front and lateral incidence after the reduction of dislocation

\section{Case 2}

The second patient was a 34-year-old right-handed computer scientist. He had painful functional impotence of the right wrist. He reported an imprecise mechanism trauma to his wrist dating back more than 5 months. He received anti-inflammatory treatment associated with immobilization and traditional massage sessions with no improvement. During examination, he complained of pain in right wrist with functional impairment. The wrist had circumferential edematous infiltration. The right hand was blocked in pronation and slightly extended (Fig. 3).

Supination was impossible without the participation of the shoulder. Flexion, active and passive extension were limited by pain. The preoperative Cooney wrist score was 50 (poor). X-ray of the right wrist in frontal and lateral incidence showed dorsal dislocation of the distal radioulna without associated fracture image (Fig. 4). After attempts with orthopedic reduction failed, surgery was performed under general anesthesia by distal resection of ulna associated with DRU arthrodesis (Sauvé-Kapandji technique). The wrist was immobilized by posterior splint.
The operative sequence was uncomplicated. Rehabilitation started at the sixth week after removal of the cast. At 6 months of follow-up, pronosupination was comparable to that of the healthy side with pseudarthrodesis stabilized (Figs. 5,6). The postoperative Cooney wrist score was 90 (excellent).

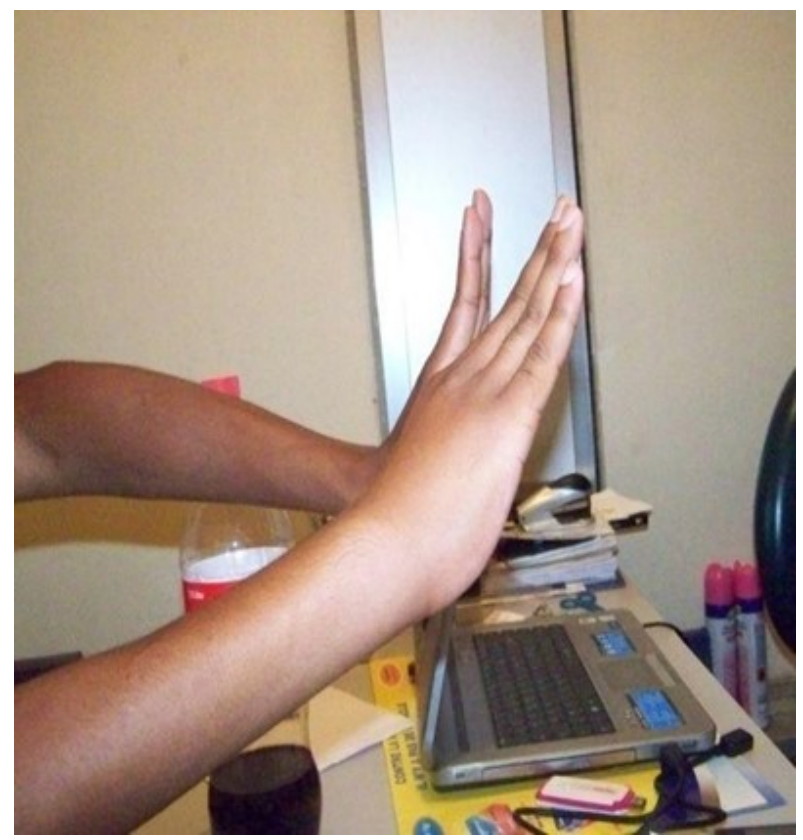

Figure 3: Functional impairment of the right wrist associated with circumferential edema.

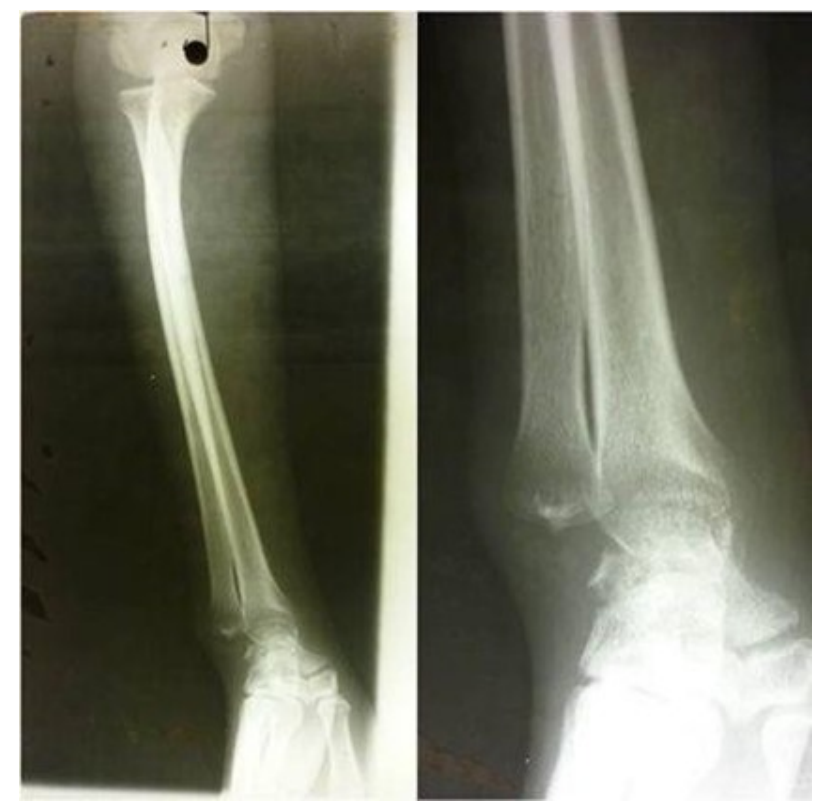

Figure 4: X-ray of the right wrist in profile incidence showing neglected dorsal dislocation of the DRU 


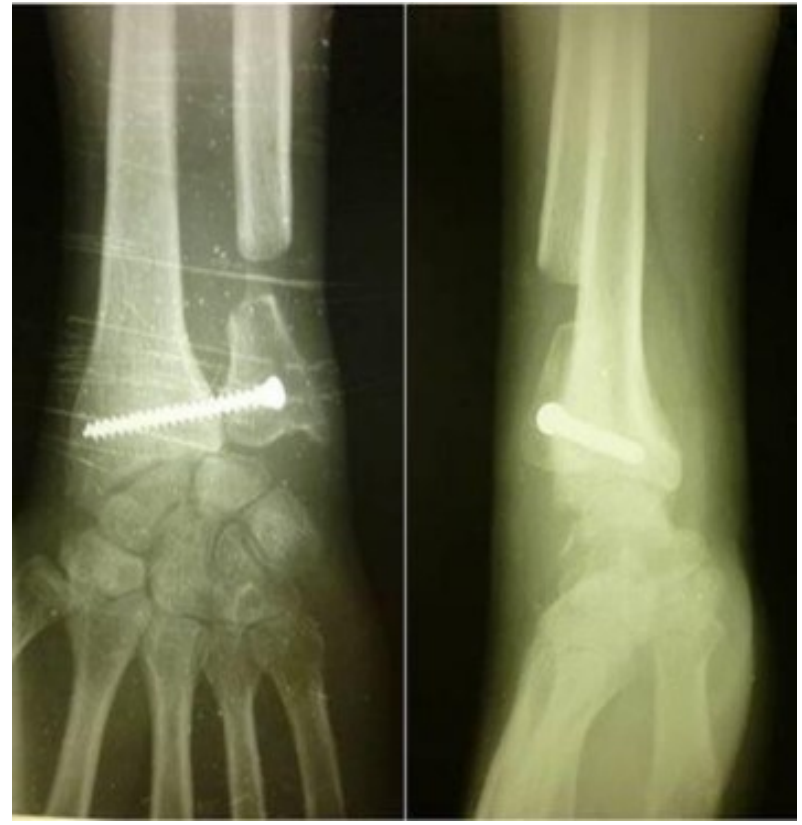

Figure 5: Right-hand wrist control x-ray in frontal and lateral incidence at 6 months of recoil showing stabilization of the pseudarthrodesis and early fusion of the DRU.

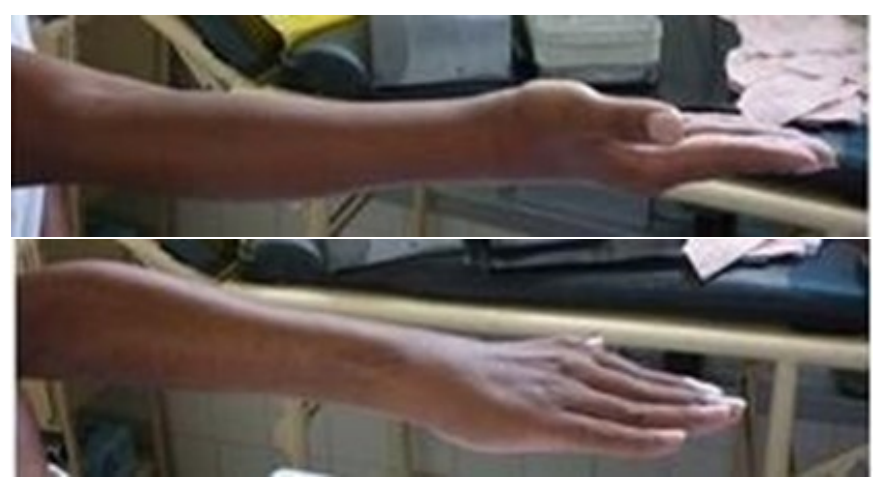

Figure 6: Clinical evaluation at 6 months postoperative with disappearance of edema, pronosupination and grip strength of the right hand.

\section{Discussion}

Distal radioulna dislocation is usually associated with fracture of the forearm bones, forming the classic Goyrand-Smith fracture. Isolated distal radioulna dislocation is rare (1). Accordingto Floares (7), the first case of distal radioulna dislocation was described by Desault in 1777 on a cadaver. Since then, occasional cases have been reported in literature. Depending on the position of the ulna head with respect to the distal radius, a dislocated DRU may be anterior (palmar) or posterior (dorsal) (3). In literature, dorsal dislocation is more common than palmar dislocation (8-9).

The mechanism of a distal radioulna dislocation is always traumatic (2). It occurs during wrist trauma in hypersupination of the forearm for palmar dislocation and hyperpronation for posterior dislocation $(8,10)$. Szabo (11) described that the head of the ulna moves forward during supination and backwards during pronation. Isolated distal radioulna dislocation passes unnoticed in more than 50\% of cases (5). Hence, most of the cases reported in literature present as distal radioulna dislocations. The signs presented on the physical examination are not obvious, such as a blocking of pronosupination, mechanical wrist pain, limitation of flexion-extension of the wrist, abnormal projection of the ulna styloid,when compared with the health side. Inspection may reveal wrist deformity with erasure of the ulna styloid (12). In both cases, an impossible supination associated with mechanical wrist pain prompted the request for an x-ray of the wrist to look for the lesion. The radiograph of the wrist face and especially comparative strict profile are usually enough to make the diagnosis. In contentious situations, a CT scan confirms diagnosis (13-14). The CT scan specifies the ratio of the ulna head with the triangular fibrocartilaginous complex. MRI and CT arthrography are of no particular interest except to eliminate carpal instability lesions (15). For our cases, the clinical examination associated with a standard x-ray of the wrist was sufficient to make the diagnosis. A radiograph of the forearm was needed to eliminate fracture of both bones.

Most recent work on distal radioulna isolated dislocations shows that the therapeutic decision depends on the evolution and complexity of the lesion (2). Usually, reduction is easy by performing a maneuver opposed to the causal mechanism associated with direct manipulation on the ulna head. In certain situations, the reduction can be blocked by incarcerating the triangular ligament or contracting the square pronator. In case of a simple and recent form, a reduction under general anesthesia followed by an immobilization plaster for 8 weeks is the choice.

Authors recommend immobilizing the forearm in pronation for palmar dislocations (16). For our first case, the forearm was immobilized in the neutral position after a pronosupination stability test.

In case of failure of orthopedic treatment, irreducibility, recurrence, and an old and complex form, surgical treatment is necessary to reduce the ulna head and repair the triangular fibro-cartilaginous complex. Several surgical techniques have been described in literature such as the Darrach procedure, the "Sauvé-Kapandji" procedure. The distal diaphyseal resection associated with a distal radioulna arthrodesis, or the Sauvé-Kapandji 
procedure (17), is our choice in cases with recurrent or old dislocations. In chronic, degenerative or delayed diagnosis, this technique seems to give satisfactory results, according to the intended functional objective (18).

\section{Conclusion}

Isolated distal radioulna dislocation is a rare lesion and often unnoticed. Wrist X-rays can sometimes be noncontributive, and a CT arthrography or magnetic resonance imaging is required to confirm the diagnosis. Management must be done urgently using surgery to avoid neglected forms.

\section{References}

1. Slattery D, Gohil S, Hogan G. A case report and the literature review: volar dislocation of the DRUJ and stabilisation using mini-suture anchors. Eur J Orthop Surg Traumatol. 2013; 23(Suppl. 2): S203- 5.

2. Caranfil R. Isolated traumatic luxation of the distal radio- ulna joint. A case report. Acta Orthop Belg. 2000; 66: 517-20.

3. Tsai P, Paksima N. The distal radioulna joint. Bull NYU Hosp Jt Dis. 2009; 67:90-6.

4. Schiller M, Ekenstam F, Kirsch P. Volar dislocation of the distal radio-ulna joint. J Bone Joint Surg Am. 1991; 73(4): 617-9.

5. Kumar A, Iqbal M. Missed Isolated Volar Dislocation of Distal Radio-ulna Joint: case report. J Emerg Med. 1999; 17(5): 873- 5.

6. Putzeys G, Scheerlinck T, Haentjens P. Isolated volar dislocation of the distal ulna at the distal radioulna joint, a case report. Acta Orthop Belg. 1999; 65(3):376 - 7 .
7. Floareş G. A new technic for the surgical treatment of old isolated dislocations of the lower end of the cubitus. Rev Med Chir Soc Med Nat Iasi. 1987; 91(1): 77- 80.

8. Singletary E. Volar dislocation of the distal radio-ulna joint. Ann Emerg. Med. 1994; 23: 881-3.

9. Rose-Innes A. Anterior dislocation of the ulna at the inferior radio ulna joint. Case report, with a discussion of the anatomy of rotation of the forearm. J Bone Joint Surg Br. 1960; 42: 515- 21.

10. Sakota J, Kaneko K, Miyahara S, et al. Luxation récidivante de la radio-ulnaire distale. À propos d'un cas. Chirurgie de la main. 2002;21: 301-4.

11. Szabo R. Distal radioulna joint instability. Instr Course Lect 2007; 56:79-89.

12. Morrissy R, Nalebuff E. Dislocation of the distal radioulna joint: anatomy and clues to prompt diagnosis. Clin Orthop Relat Res. 1979; 144:154- 8 .

13. Wechsler R, Wehbe M, Rifkin M, et al. Computed tomography in diagnosis of distal radioulna subluxation. Skeletal Radiol 1987; 16: 1- 5 .

14. Mino D, Palmer A, Levinsohn E. Radiography and computerized tomography in the diagnosis of incongruity of the distal radioulna joint. J Bone Joint Surg Am. 1985; 67: 247- 52.

15. Mittal R, Kulkarni R, Subsposh S, et al. Isolated volar dislocation of distal radioulna joint: how easy to miss. Eur J Emerg Med. 2004; 11(2): 113-6.

16. Fadi B, Mazhar F, Ayman E. Locked volar distal radioulna joint dislocation. Int J Surg Case Rep. 2016; 22: 12- 4.

17. Sauvé L., Kapandji M. Nouvelle technique de traitement chirurgical des luxations récidivantes isolées de l'extrémité inférieure du cubitus. J. Chir. 1936; 47:589- 94.

18. Kapandji IA. Opération de Sauvé-Kapandji. Techniques et indications dans les affections non rhumatis males. Ann Chir Main 1986; 5: 181 